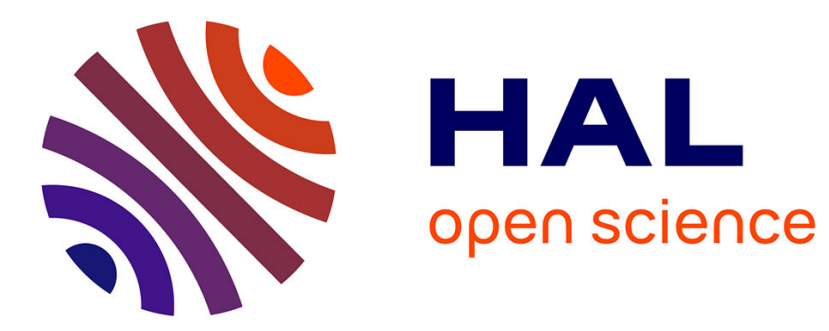

\title{
Opportune maintenance and predictive maintenance decision support
}

\author{
Edouard Thomas, Eric Levrat, Benoît Iung, Pierre Cocheteux
}

\section{To cite this version:}

Edouard Thomas, Eric Levrat, Benoît Iung, Pierre Cocheteux. Opportune maintenance and predictive maintenance decision support. 13th IFAC Symposium on Information Control Problems in Manufacturing, INCOM'2009, Jun 2009, Moscou, Russia. pp.CDROM. hal-00403887

\section{HAL Id: hal-00403887 https://hal.science/hal-00403887}

Submitted on 13 Jul 2009

HAL is a multi-disciplinary open access archive for the deposit and dissemination of scientific research documents, whether they are published or not. The documents may come from teaching and research institutions in France or abroad, or from public or private research centers.
L'archive ouverte pluridisciplinaire HAL, est destinée au dépôt et à la diffusion de documents scientifiques de niveau recherche, publiés ou non, émanant des établissements d'enseignement et de recherche français ou étrangers, des laboratoires publics ou privés. 


\title{
Opportune maintenance and predictive maintenance decision support
}

\author{
Édouard Thomas, Éric Levrat, Benoit Iung, Pierre Cocheteux \\ Centre de Recherche en Automatique de Nancy (CRAN) \\ Nancy-Université, CNRS \\ Boulevard des Aiguillettes B.P. 70239 \\ F-54506 Vandoeuvre-lès-Nancy \\ (e-mails: \{edouard.thomas, eric.levrat, benoit.iung, pierre.cocheteux\}@cran.uhp-nancy.fr)
}

\begin{abstract}
Conventional maintenance strategies on a single component are being phased out in favour of more predictive maintenance actions. These new kinds of actions are performed in order to control the global performances of the whole industrial system. They are anticipative in nature, which allows a maintenance expert to consider non-already-planned maintenance actions. Two questions naturally emerge: when to perform a predictive maintenance action; how a maintenance expert can take advantage of a given predictive maintenance action that will have to be performed. These questions concern maintenance decision making and lead to the notion of opportune maintenance actions. This new concept extends the investigation of predictive maintenance actions from one single component to several ones.
\end{abstract}

Keywords: Predictive maintenance, Maintenance engineering, Decision making, Complex systems.

\section{INTRODUCTION}

Today most conventional maintenance strategies are purely reactive, corrective (fixing or replacing equipment after it fails) or time-scheduled (Wang, 2002). These strategies are not well adapted to the control of the global performances of a manufacturing system (Gupta, et al., 2001). Therefore it is now necessary to move from traditional maintenance strategies towards condition-based maintenance performed only when a certain level of equipment deterioration occurs, or predictive maintenance which anticipates the appearance of a failure (Djurdjanovic, et al., 2003). This notion of anticipation becomes of true importance whenever a prognosis process is available (Iung, et al., 2005). Indeed the notion of anticipation allows the investigation of non-planned maintenance actions, which is part of what is called opportunistic maintenance. Opportunistic maintenance aims at answering the following questions: When to perform a particular maintenance action? What components should benefit from a preventive maintenance action? Which components should first benefit from a preventive maintenance intervention? The notion of opportunistic maintenance has been carefully reviewed in (Thomas, et al., 2008a). It was emphasised that the classical notion of opportunistic maintenance can cover up various realities and criteria. Moreover predictive vs. proactive maintenance strategies may generate numerous opportunities to perform non-previously-planned maintenance tasks. The notion of opportunistic maintenance currently does not allow to take advantage of those new opportunities-to-come, partly because of the lack of rigorous definitions and adapted formalisms of opportunistic maintenance. The aim of this paper is to propose a rigorous concept that allows a maintenance expert to make use of those generally neglected opportunities.
Therefore the definition of a new concept will be proposed and discussed in this study. The concept of opportune maintenance will be introduced in order to take advantage of the occurrence of opportunities that the new forms of predictive maintenance can anticipate. It is expected that the notion of opportune maintenance will be adapted to the challenges raised by the development of predictive maintenance strategies in some companies. The focus will be put on the adaptation of a new mathematical formalism to realistic and industrial issues.

The rest of the paper is organised as follows: the context, some new challenges due to predictive approaches in maintenance and the definition of an opportune maintenance action are presented in section 2. A discussion about the representation of the different criteria of an opportune maintenance action will be conducted in section 3. A mathematical model of those criteria will be proposed in section 4 . The issue of aggregating those criteria in order to compare two opportune maintenance actions will be discussed in section 5 , thus leading to a predictive decisionmaking tool that can be used by a maintenance expert.

\section{CHALLENGES OF PREDICIVE MAINTENANCE}

A production system is considered, and some of its components are monitored. A degradation process is supposed to be observed, at instant $t_{0}$, on one component $C$ of the system. The current instant $t_{0}$ is supposed to be situated between $T_{1}$ and $T_{2}$, where $T_{1}$ (respectively $T_{2}$ ) is the date of the last (respectively next) preventive time-scheduled maintenance action carried out on $C$. The evolution of the degradation process on $C$ is estimated by a prognosis process, which leads to a remaining useful life (RUL) of $R U L(C)$ time units if no maintenance action is performed on $C$ (Levrat, et 
$a l .$, 2008). In accordance with (ISO, 2004), a confidence area is associated to this estimation. The expected date of failure of the component $C$ is supposed to occur before $T_{2}$, even with the uncertainty associated to the estimation (Figure 1).

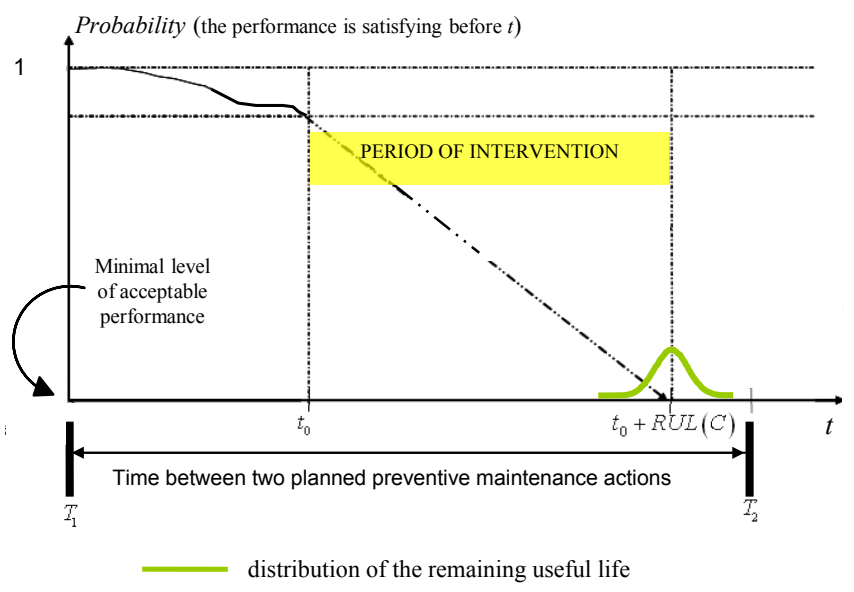

Fig. 1. Estimation of $R U L(C)$ and representation, on the same graph, of the uncertainty surrounding $R U L(C)$.

The knowledge of the temporal period that lies between the instant of the first observation of a degradation process on $C$ and the estimated date of its failure is an opportunity and can be used to plan some preventive maintenance action on $C$. This knowledge has only been made available by the use of a prognosis process. Any preventive maintenance action that will be performed on $C$ during the aforementioned temporal period will thus be a predictive maintenance action. Generally speaking, the planning of such a preventive maintenance action will lead to the following issues (Knezevic, et al., 1997): determine (1) the purpose of the action, (2) when it should be performed, (3) the nature of the action, (4) how to perform it, (5) where the components to be maintained are located, and (6) the mean time to repair (Figure 2).

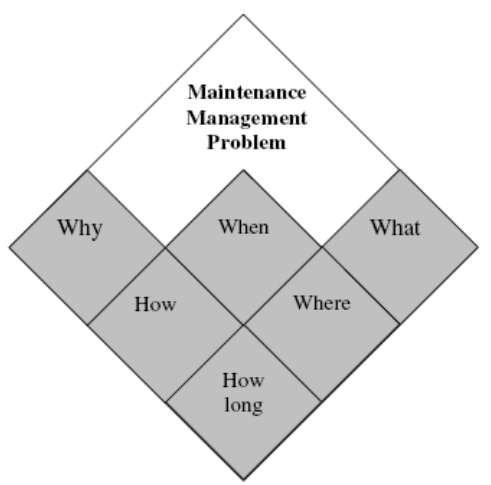

Fig. 2. Elements related to a preventive maintenance action.

In a predictive or proactive approach, the first question can be answered by making use of a diagnosis process (S.C. Thomas, et al., 1991): the occurrence of a failure (respectively: of a degradation process) on a component triggers a corrective maintenance action (respectively: the planning of a preventive maintenance action). The existence of a prognosis process addresses the third question, by evaluating the impact of different maintenance actions on the global performances of the production system (Voisin, et al., 2008). The fourth and sixth questions can be answered by using maintenance engineering, and more specifically a computerised maintenance management system that collects the information related to the different maintenance actions. Finally the second question has been carefully addressed by the authors (Levrat, et al., 2008; Thomas, et al., 2008b). The idea is to make use of the planned future production stoppages in order to carry out predictive maintenance actions before the failure of the components to be maintained. Such an approach can be viewed as opportunistic, because maintenance tries not to interfere with production. But another kind of opportunity can be addressed in an anticipative context: does the definition of a predictive maintenance action on $C$ allow the maintenance expert to investigate non-already-planned preventive maintenance actions on different degraded components? Actually, performing a predictive maintenance action on $C$ (during a production stoppage or not) can be viewed as a future opportunity to carry out some other preventive maintenance actions. Those actions could then be qualified opportunistic maintenance actions as well. Among the degraded components that would be candidates for such preventive maintenance actions, some will naturally be promoted because they are "close to $C$ ", or because they will need the same tools and skills as $C$ in order to be operated on. (Dhillon, 1999) sums up some criteria that would help promote such components: the component's location and environment; types of tools and accessories needed to perform the required tasks; clothing worn by the technical staff; specified time requirements to perform for performing the task; work clearances necessary for performing the tasks; packages of items behind the access opening (...). In order to emphasise the differences between the traditional senses of opportunistic maintenance and the new kinds of opportunities that predictive approaches allow to consider, we will refer to opportune maintenance in the following. More precisely, we propose three definitions.

Def. 1. An opportune maintenance action is a maintenance action which is carried out on a component $K$ of a production system, and which is either opportune of the first kind, or $C$ opportune of the second kind.

Def. 2. A maintenance action which is performed during a production stoppage will be opportune of the first kind.

Def. 3. A C-opportune maintenance action of the second kind is a maintenance action which is performed on a component $K$ and which satisfies at least one of the following conditions: $K$ is closed to $C$; the safety authorisations for the action on $K$ are the same as the ones for the action on $C$; a flow of matter, energy or information links $K$ to $C ; K$ and $C$ participate to the same function in the production system; the tools and skills necessary to reach $K$ are the same as the ones needed to access $C$.

The choice of the word "opportune" may be criticised, and we welcome any suggestion. However the authors wanted to emphasise that their concept is not "classical" opportunistic maintenance, in the sense that predictive strategies allow an 
expert to make use of future opportunities. Moreover a precise definition of an opportune maintenance action exists.

For the sake of simplicity, only some criteria have been considered in these definitions. The list of conditions in Def. 3 is not exhaustive, and we refer to (Thomas, et al., 2008a) for more information about the grouping of several maintenance actions. The conditions presented in Def. 3 are of physical nature (based on the structure of the system), of technical nature (based on the tools and skills needed to perform the two actions), and safety-related. They are supposed to be independent (the validation of one condition does not impact on the validation of the other conditions). They are the expression of a common property shared by both $C$ and $K$. The different conditions are now being discussed in more details.

\section{DISCUSSION ABOUT OPPORTUNE MAINTENANCE}

The rest of the document will be focused on Def. 3, as the notion of opportune maintenance actions of the first kind has already been carefully addressed and formalised by the authors (as already mentioned). The first criterion concerns the proximity of two components $K$ and $C$, the last criterion concerns the accessibility to one component from another component. As these criteria are generic and representative of the whole approach, they will be discussed in detail.

\subsection{The generic notion of proximity}

It seems that very few studies have been dedicated to the notion of proximity or closeness (Thomas, et al., 2008b). In the existing models, components are reduced to dots in a plane or a space, which is not realistic. Moreover, naive approaches such as Euclidean distances or Mahalanobis distances are not realistic as they cannot integrate the structure of the production system. Indeed two components of a same system can have very different shapes and sizes.

However one study dedicated to conception (and not to maintenance) has tackled the task of defining a realistic notion of proximity between two given components of the same production system (Rotundo and Colton, 1999). This study establishes that the parameters to take into account to promote the proximity between the components $C$ and $K$ are the physical size of $C$, the skills of the operator acting on $C$ and the tools at his disposal while acting on $C$. The authors construct three-dimensional geometric models to define a notion of proximity between $C$ and $K$. The smallest volume, encompassing $C$, that the operator needs to perform the predictive maintenance action on $C$ is called service volume and utilised to define proximity. These volumes (such as the space occupied by $C$, by a hand and by a screwdriver, or the portion of space occupied by $C$ and by an operator on a ladder) are determined by studying available tools and human factors. The approach is globally the following: let $V(C ; M)$ be the service volume associated to component $C$, with the associated predictive maintenance action $M$. Allocate, to every degraded component $K$ candidate to a preventive maintenance action $P$, a service volume $V(K ; P)$ associated to this component and to a related maintenance action. The components $K$ that are close to $C$ are, by definition, those for which some preventive maintenance action $P$ exists such that $V(C ; M) \cap V(K ; P) \neq \varnothing$. This notion of proximity makes use of the physical structure of the system, of the nature of the maintenance actions, and of the tools and skills available. However it seems a difficult task to evaluate $V(K ; P)$ where $K$ is a degraded component and $P$ a preventive maintenance action. Moreover we think that any notion of proximity between two components should only depend on the physical structure of the system, and not on the nature of a given maintenance action. The latter should be used to assess accessibility to $K$ from $C$, not proximity. Finally, with such a definition, one intuitive notion is to be lost: the notion of transitivity. It could be of interest to ensure that is $C$ is close to $K$, and if $K$ is close to $Q$, then $C$ is close to $Q$. This will be discussed in the fourth section. We now turn to the notion of accessibility.

\subsection{The notion of accessibility}

It seems that also very few studies have been dedicated to the notion of accessibility to a given component of a production system. Accessibility to a component $K$ from a component $C$ seems to be implicitly integrated (by return of experience) into the broader notion of maintainability of $K$. We refer to (Dhillon, 1999) for a discussion about the criteria affecting accessibility. The main criteria are proximity between $C$ and $K$, the tools and skills needed to reach $K$ from $C$, and the safety rules that must be satisfied when $C$ (respectively $K$ ) are being operated on. These criteria have all been taken into account in the definition of an opportune maintenance action, and will therefore be formalised in the fourth section.

\subsection{The other notions}

Every notion that has been mentioned in Def. 3 models a common property that is shared between the component $C$ (and a given predictive maintenance action associated to it) and another degraded component $K$ (and a given preventive maintenance action associated to it). For example, having the same safety rules, being accessible with the same tools, being close to one another, being linked by a flow of matter or energy, participating to the same function in the system (...) are all the expression of a common property. The mathematical tool that best allows to formalise the notion of sharing one common property is the equivalence relation. Two components will be in equivalence relation if, and only if, they share one same property. Therefore it seems that one single tool can be used to formalise all the criteria appearing in Def. 3, and thus the notion of opportune maintenance. However some concessions have to be made in order to use this single and generic mathematical tool to model all the criteria. First all the criteria need to be assessable according to the single Boolean scale $\{0 ; 1\}$ (0 if the criterion is not satisfied, 1 if the criterion is satisfied). For instance evaluations such as " $K$ is close to $C$ with probability $1 / 2$ " or " $K$ might share a given common property with $C$ " would be irrelevant. Although in this case it might be an interesting perspective to use fuzzy equivalence relations (Zadeh, 1971; Cirić, et al., 2007). Second if $K$ shares one property with $C$, 
then $C$ must share the same property with $K$. This may lead to some technical but realistic assumptions (such as the reversibility of some technical actions carried out by an operator in order to access one component, as far as the notion of accessibility is concerned). Finally the notion of transitivity must be satisfied, which might first hurt the intuition, but which is nevertheless realistic, even in the case of proximity. In the next section we propose to develop the notion of equivalence relations and to show that this simple mathematical tool is well-adapted to the concept of opportune maintenance.

\section{REPRESENTATION OF THE CRITERIA}

The first subsection is dedicated to some mathematical developments that will be used in the second subsection to formalise the notion of opportune maintenance.

\subsection{Mathematical tools}

The algebraic notion of equivalence relation is classic in both pure and applied mathematics (Sessions, 2008). Let $E$ be a finite non-empty set. An equivalence relation $\mathfrak{R}$ on $E$ is a binary relation on $E \times E$ which is reflexive, symmetric and transitive. More precisely, $\mathfrak{R}$ associates to one element $x$ of $E$ one element $y$ of $E$ (binary relation). Whenever $y$ is associated to $x$, we note $x \mathfrak{R y}$. Every element $x$ of $E$ is associated to itself: $\forall x \in E, \quad x \Re x$ holds (reflexivity). Whenever $x$ is associated to $y$ for $\mathfrak{R}, y$ is associated to $x$ : $\forall(x ; y) \in E \times E, \quad x \Re y \Rightarrow y \Re x \quad$ holds (symmetry). Whenever $x$ is associated to $y$ and $y$ is associated to $z, x$ is associated to $z$ (transitivity): $\forall(x ; y ; z) \in E \times E \times E, \quad(x \Re y$ and $y \mathfrak{R} z) \Rightarrow x \mathfrak{R}$. This defines the equivalence relation $\mathfrak{R}$.

The equivalence class $\mathfrak{R}(x)$ of an element $x$ of $E$ for the equivalence relation $\mathfrak{R}$ on $E$ is the set of all the elements in $E$ that are in relation with $x: \mathfrak{R}(x):=\{y \in E \mid x \mathfrak{R} y\}$.

The following facts are elementary: the equivalence class $\mathfrak{R}(x)$ of $x$ in $E$ cannot be empty, as $x$ lies in $\mathfrak{R}(x)$. Conversely, every element $x$ in $E$ belongs to one equivalence class, which is $\mathfrak{R}(x)$. For $x$ and $y$ two elements in $E$, we have $\mathfrak{R}(x)=\mathfrak{R}(y)$ if, and only if, $x \mathfrak{R} y$. In conclusion, the set of all the equivalence classes on $E$ defines a partition of $E$. We now turn to the formalisation of opportune maintenance.

\subsection{Application to the definition of opportune maintenance}

First a formalisation of the notion of proximity is proposed. The production system will be assimilated to the finite set of its constituting components. Computer-aided design softwares such as Catia or SolidWorks provide, in a given fixed coordinate system, some geometric characteristics for the components that have been modelled. Let $K$ and $C$ be two such components of the system. Axis-aligned bounding boxes encompassing these elements will be used. Such a box is in fact a parallelepiped in the axes of the coordinate system and includes the whole corresponding element. These bounding boxes are automatically generated by the software. Figures 3 represents two two-dimensional bounding boxes within the framework of a computer-aided design model: one bounding box $B_{K}$ encircling the element $K$, and one bounding box $B_{C}$ bordering the element $C$. The two bounding boxes meet.

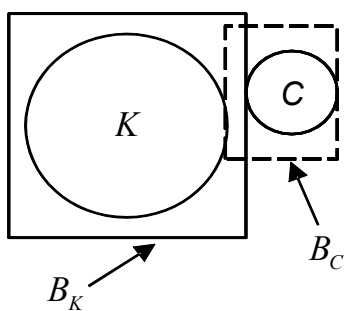

Fig. 3. Two bounding boxes with non empty intersection.

Equivalence relations will be used to formalise the different criteria occurring in Def. 3, and not the concept of opportunity itself. Here, the two components $C$ and $K$ are in relation of proximity if there exists a non negative number $n(C ; K)$ of components $Q_{1}, Q_{2} \ldots Q_{n(C ; K)}$ such that the bounding box of $\mathrm{C}$ meets the bounding box of $Q_{1}$, and the bounding box of $Q_{1}$ meets the bounding box of $Q_{2}$, and... and the bounding box of $Q_{n(C ; K)}$ meets the bounding box of $K$. As an example, Figure 4 illustrates the relation of proximity with five different components $C_{1}, C_{2}, C_{3}, C_{4}$ and $C_{5}$.

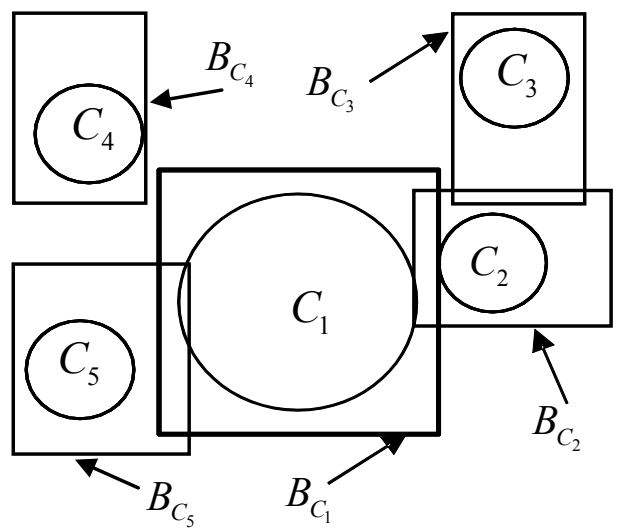

Fig. 4. Five different components and their related axisaligned bounding boxes.

The definition of the relation of proximity is illustrated on Figure 4. The two components $C_{3}$ and $C_{5}$ are in relation of proximity (consider $n\left(C_{3} ; C_{5}\right)=2$ ) and $Q_{1}=C_{2}, Q_{2}=C_{1}$ in the definition). The only component $C_{4}$ is in relation of proximity with is $C_{4}$ itself. It is very easy to see that the proposed definition of the relation of proximity is an equivalence relation. With the help of some computer-aided design software, proximity between $K$ and $C$ can be $a$ priori decided.

The second criterion that will be formalised is accessibility. An enlarged toolbox associated to a given operator is defined as the tools that every maintenance operator has at his disposal, and his own specific skills which result in his ability 
to make use of some specific devices. The enlarge toolbox reflects the skills, authorisations and abilities of a maintenance operator. The accessibility to a component $K$ from a component $C$ can then be defined. $K$ and $C$ are in relation of accessibility by one given maintenance operator if there exists a set of tools in the operator's enlarged toolbox such that allows him to reach $K$ from $C$. This definition of the relation of accessibility is currently being developed and clarified. It depends on the maintenance operator. And if it is supposed that every operation is reversible, then it is easy to see that, for every maintenance operator, the relation of accessibility is an equivalence relation. It should be stressed that maintenance engineering can, in theory, provide the list of basic vs. specific tools needed to reach the component $K$ from the component $C$. Therefore accessibility to $K$ from $C$ by one given maintenance operator can be a priori decided.

The next criterion to be investigated is the notion of safety. For the sake of simplicity, it will be assumed that the safety requirements on a component, expressed in terms of lock out - tag out, are the same during the whole maintenance operation on that component. Actually three phases have to be considered when performing a maintenance action (Levin, 1951; Duffuaa, et al., 1998): the preparation phase, the execution phase, and the closing phase. This distinction would lead to three complementary definitions. Without loss of generality, we will suppose, for expository sake, that only one phase (and therefore one definition) is considered. Two components $K$ and $C$ with two respective associate maintenance actions will be in relation of safety if the lock out - tag out required to perform the two maintenance actions are the same. This definition clearly is an equivalence relation. It enables to take into account the safety of the operator, of the system and of the environment. Legal recommendations and requirements enable a maintenance expert to decide a priori whether two components (with related maintenance actions) are in relation of safety or not.

Finally the other criteria can be formalised the same way: the property of participating to the same function within the production system defines an equivalence relation. The property of exchanging one flow (of any kind) defines an equivalence relation on the set of components of the production system. A maintenance expert can decide a priori whether two components share one of these properties or not. Therefore all the criteria can be formalised by equivalence relations. In conclusion, the notion of opportune maintenance can be modelled by one single mathematical tool. The next section proposes to aggregate all these equivalence relations in order to "quantify" the notion of opportune maintenance.

\section{AGGREGATION OF THE DIFFERENT CRITERIA}

The formalisation of opportune maintenance involves several equivalence relations. Once a component $C$ undergoing a degradation process has been identified, and once a predictive maintenance action has been decided to restore the global performances of the production system, $C$-opportune maintenance actions of the second kind can be investigated. Every preventive maintenance action on any component of the production system is potentially an eligible candidate. As soon as $C$ and a predictive maintenance action are identified, the different criteria in Def. 3 can be decided for both one degraded component $K$ and a preventive maintenance action. Therefore, theoretically speaking, all the possible $C$ opportune maintenance actions could be known short after the diagnosis of $C$ and the decision to perform a predictive maintenance action on this component. One natural question is thus: if several $C$-opportune maintenance actions of the second kind are candidates, how could a maintenance expert select the "most appropriate" one(s)? This question naturally leads to the definition of an aggregation function of the different criteria. In this subsection, the study of such an aggregation function is proposed. First, the different criteria in Def. 3 were supposed to be independent: they do not interact. Then the criteria can all be evaluated according to one common Boolean scale $\{0 ; 1\}$ : a criterion is either satisfied, or not. Finally the aggregation operator needs to satisfy some hypotheses. Let $n$ be the number of Boolean criteria, $x_{1} \ldots x_{n}$ the Boolean variables, and $F$ the aggregation function. Without loss of generality, we will suppose $F:\{0 ; 1\}^{n} \rightarrow[0 ; 1]$, with the following conditions:

- $F$ is symmetric: $F\left(x_{\sigma(1)} ; \ldots ; x_{\sigma(n)}\right)=F\left(x_{1} ; \ldots ; x_{n}\right)$ for every permutation $\sigma$,

- $F$ is idempotent: $F(0 ; \ldots ; 0)=0$ and $F(1 ; \ldots ; 1)=1$,

- $F\left(1-x_{1} ; \ldots ; 1-x_{n}\right)=1-F\left(x_{1} ; \ldots ; x_{n}\right)$,

- $F$ is increasing: $x_{\mathrm{i}}{ }^{\prime} \geq x_{\mathrm{i}}$ for every $i$ in $\{1 ; \ldots ; n\}$ implies $F\left(x_{1}{ }^{\prime} ; \ldots ; x_{n}{ }^{\prime}\right) \geq F\left(x_{1} ; \ldots ; x_{n}\right)$, with strict inequality if there exists one index $j$ in $\{1 ; \ldots ; n\}$ such that $x_{j}^{\prime}=1$ and $x_{j}=0$.

The first condition means that the order of the criteria does not interfere on the result of the aggregation process. The second condition states first that a preventive maintenance action on one component that does not share one single property with $C$ (and the related predictive maintenance action) cannot be considered a $C$-opportune maintenance action of the second kind. Then it states that a preventive maintenance action on one component that does share all the fixed properties with $C$ is "as $C$-opportune of the second kind as possible". The third condition is related to the way $F$ is evaluated: the Boolean evaluation scale $\{0 ; 1\}$ can be reversed. The fourth condition means that sharing one more property with $C$ and the predictive maintenance action related to $C$ does not lower the global evaluation.

Such functions do exist: consider for example the arithmetic mean of the Boolean variables. The issue is to get all the possible aggregation functions satisfying the aforementioned four properties. Unfortunately, it seems that all the results related to this problem consider continuous functions, i.e. they suppose at least that $F$ is defined on $[0 ; 1]$ instead of \{0;1\} (Aczél, 1948; Aczél, 1966; Aczél and Dhombres, 1989; Bustince, et al., 2007). Therefore a study of such functions by the authors and experts has been undertaken, and the conclusions are still under review at the time of writing the present article. It might be that the aggregation functions $F$ could be characterised, at least if the number $n$ of arguments is low $(n \leq 10)$, but it should be stressed that the arithmetic mean will not be the only solution. 
Indeed, in the case where $n=3$, the function $F$ defined by: $F\left(x_{1} ; x_{2} ; x_{3}\right):=c\left(x_{1}+x_{2}+x_{3}\right)+(1-3 c) \cdot\left(x_{1} x_{2}+x_{1} x_{3}+x_{2} x_{3}-2 x_{1} x_{2} x_{3}\right)$

satisfies the four aforementioned properties for $c$ in $] 0 ; 1 / 2[$. Remark that, for $c=1 / 3, F$ is the arithmetic mean.

The conclusion is that such aggregation functions do exist. Any convenient $F$ can be used by a maintenance expert as a maintenance decision-making tool to quantify "how much opportune" a $C$-opportune maintenance action of the second kind is. $F$ can therefore be used to compare $C$-opportune maintenance actions of the second kind in order to select, among the candidates, those for which the aggregation function is the largest. For these privileged components, the maintenance expert can decide to perform a non-alreadyplanned preventive maintenance action: these components are the best-placed (in sense of Def. 3) to benefit from a maintenance action. An industrial application on TELMA platform in Nancy is currently in development.

\section{CONCLUSION}

Predictive maintenance raises new challenges and allows a maintenance expert to investigate and make use of opportunities that could not be exploited before. The notion of opportune maintenance actions was introduced, rigorously defined and discussed, in order to reflect that it now becomes possible, to maintenance purpose, to consider opportunities that are generally ignored. Academic examples have been proposed to illustrate how such opportunities may be taken advantage of.

\section{ACKNOWLEDGEMENTS}

The authors would like to thank Jean-Luc Marichal for proposing the non trivial aggregation function in the case $n=3$, Pierre Marchand for helping in the study of aggregation functions, and a referee for interesting questions.

\section{REFERENCES}

Aczél, J. (1948). On mean values. Bulletin of the American Mathematical Society, 54, 392-400.

Aczél, J. (1966). Lectures on functional equations and their applications. Academic Press Incorporated, New York, 410 pages.

Aczél, J. and J. Dhombres (1989). Functional equations in several variables. Cambridge University Press, Cambridge, 464 pages.

Bustince, H., J. Montero, E. Barrenechea and M. Pagola (2007). Semiautoduality in a restricted family of aggregation operators. Fuzzy Sets and Systems, 158 (12), 1360-1377.

Ćirić, M., J. Ignjatović and S. Bogdanović (2007). Fuzzy equivalence relations and their equivalence classes. Fuzzy Sets and Systems, 158 (12), 1295-1313.

Dhillon, B.S. (1999). Engineering maintainability. How to design for reliability and easy maintenance. Gulf Professional Publishing, Houston (Texas), 254 pages, Chapter 5.
Djurdjanovic, D., J. Lee and J. Ni (2003). Watchdog Agent -an infotronics-based prognostics approach for product performance degradation assessment and prediction. Advanced Engineering Informatics, 17, 109-125.

Duffuaa, S. O., A. Raouf and J. Campbell (1998). Planning and control of maintenance systems. Wiley, New York, 400 pages.

Gupta, D., Y. Günalay and M.M. Srinivasan (2001). The relationship between preventive maintenance and manufacturing system performance. European Journal of Operational Research, 132, 146-162.

ISO 13381-1 (2004). Condition monitoring and diagnostics of machines - Prognostics. International Standard Organization.

Iung, B., M. Véron, M.-C. Suhner and A. Muller (2005). Integration of maintenance strategies into prognosis process to decision-making aid on system operation. Annals of the CIRP, 54 (1), 5-8.

Knezevic, J., L. Papic and B. Vasic (1997). Sources of fuzziness in vehicle maintenance management. Journal of Quality in Maintenance Engineering, 3 (4), 281-288.

Levrat, E., E. Thomas and B. Iung (2008). Odds-based decision-making tool for opportunistic productionmaintenance synchronization. International Journal of Production Research, 46 (19), 5263-5287.

Lewin, K. (1951). Field theory in social science: selected theoretical papers. Cartwright, D. (Editor), Harper and Row, New York, 346 pages.

Rotundo, V. and J.S. Colton (1999). Serviceability considerations for the layout of coiled tubing units. Journal of Engineering Design, 10 (3), 259-275.

Sessions, R. (2008). Simple architectures for complex enterprises. Microsoft Press, Redmond (Washington), 208 pages, Chapter 3.

Thomas, E., E. Levrat and B. Iung (2008a). Overview on opportunistic maintenance. In: Proceedings of the $9^{\text {th }}$ IFAC Workshop on Intelligent Manufacturing Systems, $9^{\text {th }}-10^{\text {th }}$ October 2008, Szczecin, Poland.

Thomas, E., E. Levrat, and B. Iung (2008b). Maintenance decision-making tool reaching a compromise between maintainability and reliability performances. In: Proceedings of the $17^{\text {th }}$ IFAC World Congress, $6^{\text {th }}-11^{\text {th }}$ July 2008, Seoul, Republic of Korea.

Thomas, S.C., D.P. Gaver and P.A. Jacobs (1991). Inspection models and their application. IMA Journal of Mathematics Applied in Business and Industry, 3 (4), 283-304.

Voisin, A., E. Levrat, P. Cocheteux and B. Iung (2008). Generic prognosis model for proactive maintenance decision support: application to pre-industrial emaintenance test bed. Journal of Intelligent Manufacturing, DOI 10.1007/s10845-008-0196-z.

Wang, H (2002). A survey of maintenance policies of deteriorating systems. European Journal of Operational Research, 139, 469-489.

Zadeh, L.A. (1971). Similarity relations and fuzzy orderings. Information Sciences, 3 (2), 177-200. 